\title{
Comparison of Theoretical and Experimental Bending and Torsional Moments of Endodontic Files and Reamers
}

R. G. CRAIG, E. D. MC ILWAIN, and F. A. PEYTON

School of Dentistry, University of Michigan, Ann Arbor, Michigan

A number of researchers have reported experimental bending ${ }^{1-4}$ and torsional ${ }^{5}$ moment curves for endodontic files and reamers. We recently reported a study in which bending and torsional moments versus angular deflection were determined by an improved method in which highly sensitive torquemeters were used. ${ }^{6}$

Timoshenko ${ }^{7}$ and Papov ${ }^{8}$ present formulas for bending and torsion of beams, based on Hooke's Law, in which the stress is proportional to strain. These formulas apply to the elastic behavior of beams and cannot be applied in the plastic range. $\mathrm{Nadai}^{9} \mathrm{de}^{-}$ scribes the ideal behavior of a completely plastic material. It is not possible to describe plastic behavior accurately in general since few materials approach ideal behavior. With flexural and torsional formulas as a guide, however, approximations of plastic behavior can be described for many materials.

It is the purpose of this paper to describe the simple flexural and torsional formulas found in textbooks on strength of materials and to compare calculated bending and torsional moments obtained using these equations with experimental values. Reasonable agreement of calculated and experimental values would indicate an opportunity for design of endodontic instruments with specified bending and torsional properties.

FLEXURE FORMULA.-The flexure formula $^{8}$ describes the bending of a straight beam with constant cross section and an axis of symmetry in which the bending moments lie in a plane containing the axis of symmetry and the axis of the beam (Fig. 1 ). When the beam is bent, one side is in tension and the other side is in compression.

This investigation was supported by USPHS Research Grant DE.01234 from the National Institute of Dental Research, National Institutes of Health, Bethesda, Md.

Received for publication January 13, 1967.
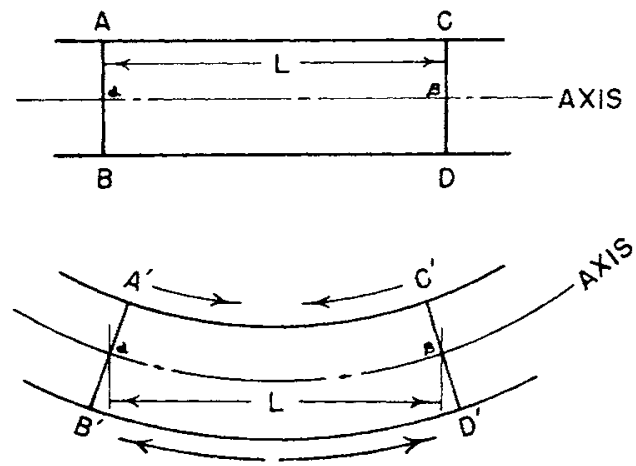

FIG. 1.-A straight beam with a constant cross section and an axis of symmetry in which the bending moments lie in a plane containing the axis of symmetry and the axis of the beam.

However, a plane section through the beam, taken normal to the axis, $\mathrm{AB}$ or $\mathrm{CD}$, remains plane after the beam is bent, $A^{\prime} B^{\prime}$ or $\mathrm{C}^{\prime} \mathrm{D}^{\prime}$. This condition applies in both elastic and plastic behavior. In each plane, there exists a point $a$ or $\beta$, which is not stressed, and the collection of all such points forms the neutral surface of the beam. This surface lies between the area of tensile and compressive stresses. In the elastic region, the neutral surface passes through the centroid of the cross-sectional area of the beam. In the plastic region, the location of the neutral surface depends on the comparative tensile and compressive properties of the material. If the tensile and compressive properties are identical, the neutral surface will remain through the centroid.

In a beam subjected to bending, strains in the fibers vary linearly as their distances from the neutral surface. The axial strains are associated with stresses that act normal to the section of a beam (elastic or plastic). On the section of a beam, normal stresses resulting from the bending vary linearly as 
their distance from the neutral surface (elastic only).

The flexure formula is derived from the foregoing discussion and is as follows:

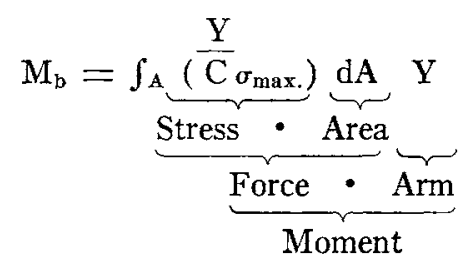

Where $M_{b}$ is the bending moment, $Y$ is the distance from the neutral surface to the point, $\mathrm{C}$ is the distance from the neutral surface to the most remote point, and $\sigma$ is the stress.

Equation 1 can be written as

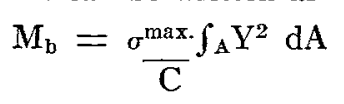

However,

$$
\int_{\mathrm{A}} \mathrm{Y}^{2} \mathrm{dA}=\mathrm{I}
$$

where $I$ is the moment of inertia about the neutral axis.

Equation 2 can be written as

$$
\mathrm{M}_{\mathrm{b}}=\frac{\sigma_{\mathrm{C}}^{\max } \cdot \mathrm{I}}{\overline{\mathrm{C}}}
$$

The section modulus of the beam, $Z$, equals $1 / 0$; therefore, equation 4 may be simply expressed as

$$
\mathrm{M}_{\mathrm{b}}=\sigma_{\text {max. }} \mathrm{Z}
$$

Equation 5 is the flexure formula that holds only for elastic behavior, or the highest usable value for $M_{b}$ is the yield point moment. If the ultimate bending moment, $\mathrm{M}_{b}$ ult., was used, and from it $\sigma$ max. was calculated, the "rupture modulus," $M_{b}$ ult./ $Z$, would be obtained. The "rupture modulus" always exceeds the true stress reached in a material at failure and is about 50 percent greater when rectangular cross sections are considered.

The ultimate bending moment, $\mathrm{M}_{\mathrm{b}}$ ult., is used sometimes with respect to a beam's load-bearing capacity. A useful approximation, has been used to describe bending behavior in the fully elastic range. ${ }^{7,9}$

$$
M_{b} \text { ult. } \simeq 3 / 2 M_{b} \text { yield point }
$$

Thus, by means of the "rupture modulus" or the yield point-bending moment, plastic

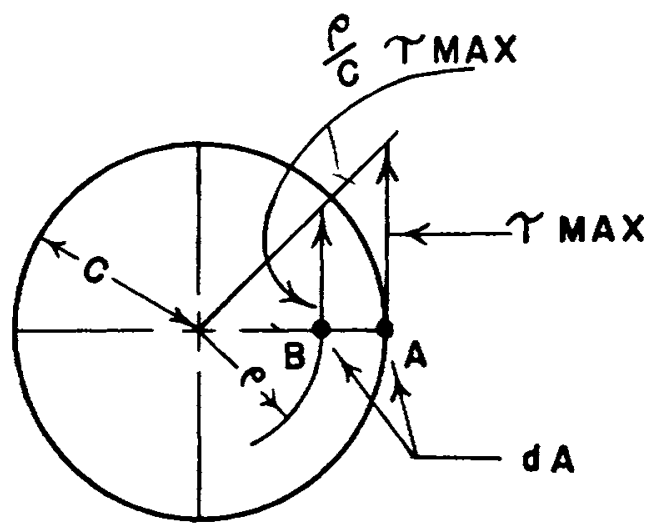

FIG. 2-Torsion of a straight rod with a circular cross section.

behavior in bending may be approximated.

TORSION FORMULA.- The torsion formula ${ }^{8}$ describes torsion in the elastic range and applies to straight members of circular cross section (Fig. 2). It is derived on the assumptions that, first, a plane section of material perpendicular to the axis of the member remains plane after torque is applied, so that no warping or distortion of parallel planes perpendicular to the axis of the member takes place. Second, the shearing strains vary linearly from the central axis. Third, the stresses induced by the assumed distortions are shearing stresses and lie in a plane parallel to the section taken normal to the axis of the member, that is not axialy loaded. The torsional moment, $M_{t}$, is derived on this basis and is expressed as

$$
\mathrm{M}_{\mathrm{t}}=\int_{\mathrm{A}}\left(\frac{\rho}{\mathrm{C}} \tau_{\max }\right) \mathrm{dA} \rho
$$

where $\rho$ is the distance from the axis to the point, $\mathrm{C}$ is the distance from the axis to the most remote point and $\tau$ is the shearing stress.

Since

$$
\int_{\mathrm{A}} \rho^{2} \mathrm{dA}=\mathrm{J}
$$

where $J$ is the polar moment of inertia about the axis, equation 7 can be written as

$$
\mathrm{M}_{\mathrm{t}}=\frac{\tau_{\mathrm{C}}^{\max } \mathrm{J}}{\mathrm{C}}
$$

The polar section modulus $Z_{\mathrm{p}}$ equals $\mathrm{J} / \mathrm{c}$ and therefore,

$$
\mathrm{M}_{\mathrm{t}}=\tau \max . Z_{\mathrm{p}}
$$



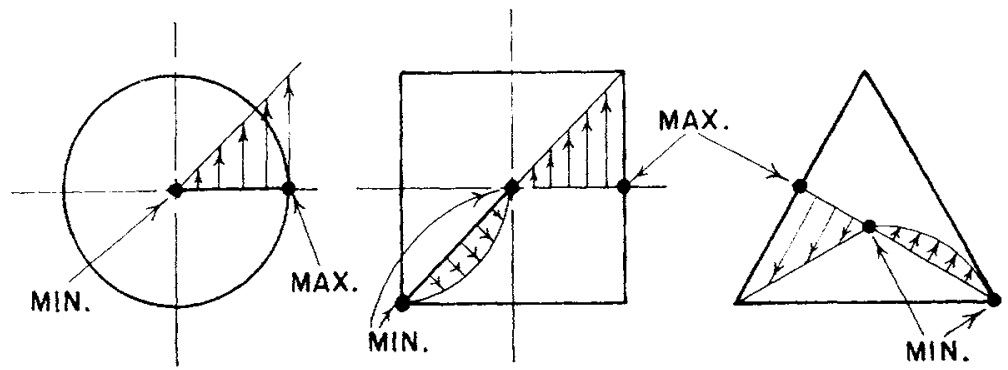

FIG. 3.-Diagrams illustrating shearing stresses in variously shaped cross sections.
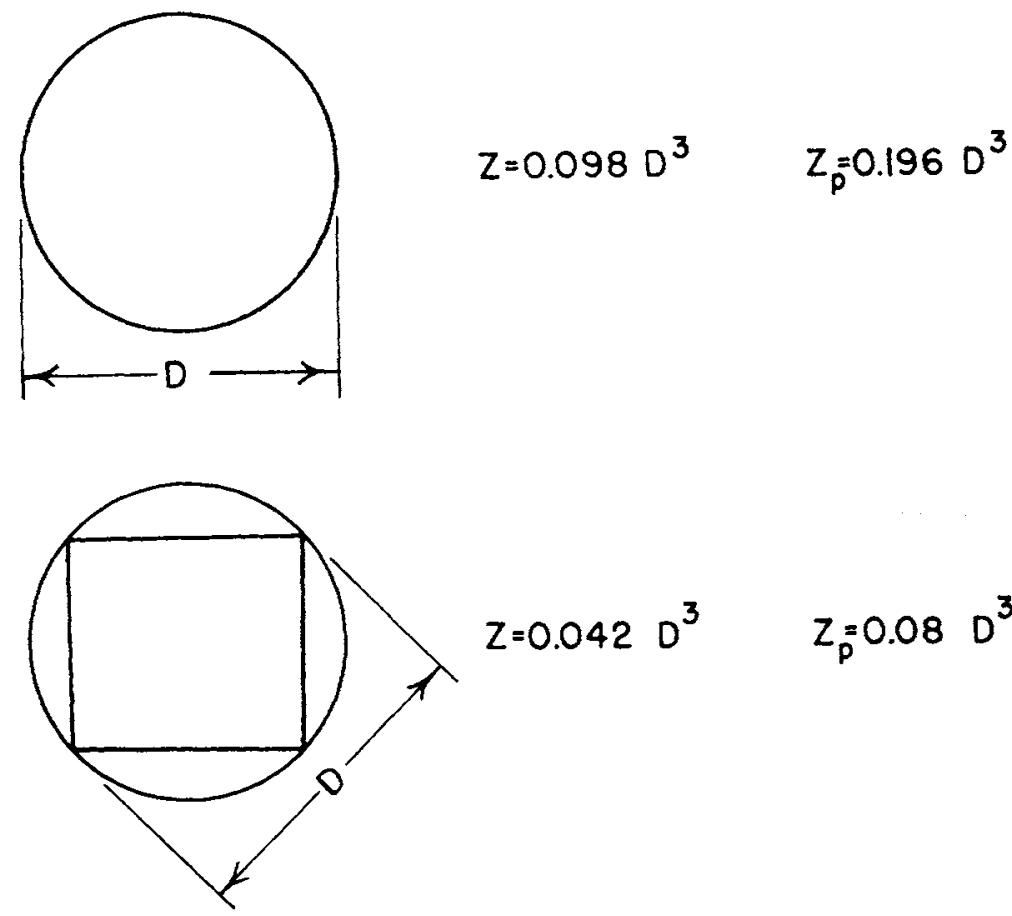

$Z=0.042 D^{3}$

$Z_{p}=0.08 D^{3}$

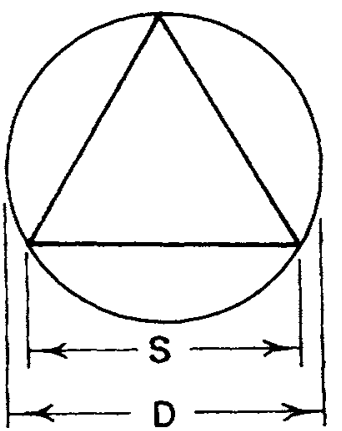

$$
\begin{aligned}
Z & =0.031 \mathrm{~s}^{3} \\
& =0.020 \mathrm{D}^{3}
\end{aligned}
$$$$
z_{\bar{p}} 0.05 \mathrm{~s}^{3}
$$$$
=0.030^{3}
$$

FIG. 4.-Section moduli, $Z$, and polar section moduli, $Z_{\mathrm{p}}$, formulas for circular, square, and triangular cross sections.

\section{NOTE: $\quad z_{p} \simeq 2 Z$}


Equation 10 is the torsion formula that holds for the elastic range. Beyond the elastic range, variations of strain from the center to outer edge remain linear but, beyond the yield point, shearing stress does not vary linearly. Again $\mathbf{M}_{\mathrm{t}}$ ult. can be used to calculate $\tau_{\max }$. and obtain the "rupture modulus," $M_{t}$ ult. $/ Z_{p}$. The "rupture modulus" always exceeds the true stress in the material at failure, but it functions as a rough index of the ultimate torsional strength of the material. The following experimentally determined relation can be used to describe the behavior beyond the elastic range. ${ }^{7}$

$$
\mathrm{M}_{\mathrm{t}} \text { ult. } \simeq 4 / 3 \mathrm{M}_{\mathrm{t}} \text { yield point }
$$

In rectangular and triangular sections, the corner elements do not distort. Shearing stresses are zero at the corners and are maximum at the midpoint of the longest sides. ${ }^{7,8}$ The diagrams in Figure 3 illustrate the stresses in various cross sections used in endodontic instruments. The plastic behavior in torsion may be approximated using the "rupture modulus" or the yield point-torcinnal moment lus values will be used to calculate moments the formulas are given in Figure $4 .{ }^{10} \mathrm{It}$ should be noted that the polar section modulus values approximate twice the values of the section modulus.

\section{Materials and Methods}

Bending and torsional moment versus angular deflection curves were obtained by using a series of torque meters with ranges of 0.05 to 25 inch-ounces which permitted the determination of the bending and torsional moments. The test methods were described in a previous study. ${ }^{6}$

The yield point and ultimate bending and torsional moments reported in the present study also were obtained from the moment versus angular deflection curves in the previous study. ${ }^{6}$

\section{Results}

The ultimate bending and torsional moments of carbon steel endodontic instruments were determined by equations 6 and 11 , and yield point moments were obtained from experimental bending moment-deflectinn rurves ${ }^{6}$ The ralculated $M_{n}$ ult values 
TABLE 2

Rupture Moduli of Conventional Carbon Steel Endodontic Instruments

\begin{tabular}{|c|c|c|c|c|c|c|c|c|}
\hline \multirow[b]{2}{*}{$\begin{array}{l}\text { Instrument } \\
\text { No.* }\end{array}$} & \multirow[b]{2}{*}{$\begin{array}{c}\mathrm{d} \\
\text { At } 3 \mathrm{~mm} \\
\text { (imch } \times 10^{-4} \text { ) }\end{array}$} & \multirow[b]{2}{*}{$\begin{array}{c}\mathrm{d}^{3} \\
\left(\text { (inch }^{3}\right. \\
\left.\times 10^{-7}\right)\end{array}$} & \multicolumn{3}{|c|}{ Bending } & \multicolumn{3}{|c|}{ Torsion } \\
\hline & & & $\begin{array}{c}\mathrm{Z} \\
\left(\mathrm{inch}^{8}\right. \\
\left.\times 10^{-8}\right)\end{array}$ & $\begin{array}{l}\text { Mbult. } \\
\text { (in. }-1 \mathrm{~b} . \\
\times 10^{-2} \text { ) }\end{array}$ & $\begin{array}{l}\text { or.m. } \\
\text { (psi } \\
\left.\times 10^{5}\right)\end{array}$ & $\begin{array}{l}Z_{p} \\
\left(\text { inch }^{3}\right. \\
\times 10^{-8} \text { ) }\end{array}$ & $\begin{array}{l}\mathrm{M}_{\mathrm{t}} \text { ult. } \\
\text { (in.-1b. } \\
\times 10^{-2} \text { ) }\end{array}$ & $\begin{array}{r}\tau \mathrm{r} . \mathrm{m} . \\
(\mathrm{psi} \\
\left.\times 10^{5}\right)\end{array}$ \\
\hline F $1+$ & 63 & 2.5 & 1.0 & 0.63 & 6.3 & 2.0 & 0.94 & 4.7 \\
\hline F $2 \div$ & 87 & 6.8 & 2.6 & 1.9 & 7.3 & 5.2 & 1.9 & 3.7 \\
\hline F $3 t$ & 114 & 15 & 6.0 & 5.6 & 9.3 & 12 & 4.4 & 3.8 \\
\hline F $4 t$ & 153 & 36 & 14 & 16 & 11.4 & 28 & 12.5 & 4.5 \\
\hline $\mathrm{F} 5 \dagger$ & 193 & 72 & 29 & 28 & 9.7 & 58 & 19 & 3.3 \\
\hline $\mathrm{F} 6^{\dagger}$ & 236 & 130 & 52 & 50 & 9.6 & 100 & 34 & 3.4 \\
\hline R $1 \dagger$ & 79 & 5.0 & 2.0 & 1.5 & 7.5 & 4.0 & 0.94 & 2.4 \\
\hline R $2 \dagger$ & 98 & 9.5 & 3.8 & 3.1 & 8.2 & 7.8 & 2.5 & 3.2 \\
\hline R 3t & 118 & 16 & 4.8 & 3.1 & 6.5 & 8.0 & 1.9 & 2.4 \\
\hline R $4 t$ & 161 & 42 & 13 & 9.4 & 7.2 & 21 & 4.1 & 2.0 \\
\hline R 57 & 193 & 72 & 22 & 15 & 6.8 & 36 & 9.4 & 2.6 \\
\hline R $6 \ddagger$ & 228 & 120 & 36 & 25 & 7.0 & 60 & 15 & 2.5 \\
\hline
\end{tabular}

$* \mathrm{~F}=$ file; $\mathrm{R}=$ reamer

†Square cross-sectional configuration

tTriangular cross-sectional configuration.

moment values obtained from experimental curves are listed in Table 1. Comparison of the calculated and observed ultimate moments provides an estimate of the reliability of the equations in this application. The agreement in general is good, but closer agreement was obtained with instruments smaller than no. 5. It appears, however, that the ultimate bending moment can be predicted with reasonable accuracy when the yield point moment is known.

The calculation of $M_{b}$ ult. from $M_{b}$, y.p is only possible if the moment-deflection curves up to and just beyond the yield point are determined. The formulas for the "rupture moduli" are derived from theoretical considerations, and therefore give a better indication of how ideal the behavior of the files and reamers are in bending and torsion.

The cross section of the instruments was considered to be uniform and to have the value observed $3 \mathrm{~mm}$. from the tip. The values for $d$ at $3 \mathrm{~mm}$. are listed in Table 2 ; the calculated section moduli for the various instruments are based on these values and the cross-sectional shape of the instrument. Also listed in Table 2 are the $\mathrm{M}_{\mathrm{r}}$ ult. and $M_{t}$ ult. values in inch-pounds and the calculated rupture moduli in bending, or.m., and torsion, $\tau$ r.m.

\section{Discussion}

The observed values of $M_{b, s . p .}, M_{b} u l t$, $M_{t, y . p .}$, and $M_{t}$ ult. listed in Table 1 are means taken from three bending or torsional moment-angular deflection curves. If the calculated and observed $M_{t}$ ult. values are summed, and grand means $\overline{\bar{x}}_{c}$ and $\overline{\bar{x}}_{p}$ are obtained as shown in Table 1 , the $t$ test for the difference between these means can be used to test the hypothesis that the means are the same and indicates whether there was a difference between the observed and calculated values of the ultimate bending moments for files and reamers. A $t_{(22)}$ of 1.32 showed that the means were the same at the 95 percent confidence level.

A similar $t$ test was run for the calculated and observed torsional moments and a $t_{(22)}$ of 0.20 again showed that the means were same at the 95 percent confidence level. The evidence is that the ultimate bending or torsional moment can be reliably estimated from the corresponding yield point-bending or torsional moment.

If the instruments functioned ideally, or.m. and $\tau$ r.m. should approximate the tensile strength and shear strength, respectively, of the carbon steel music wire. The means of the values of or.m. for files and the reamers are 893,000 and 720,000 psi, respectively, which are much higher than the tensile strength of 330,000 psi. Even a correction for the "rupture modulus" being 50 percent higher than the observed tensile strength gives values of 596,000 and 480,000 psi, which still are high compared with those for the tensile strength.

The means for $\tau \mathrm{r} . \mathrm{m}$. for the files and reamers were 390,000 and 252,00 psi, respectively. These means, particularly the means for the reamers, agree quite well with 
the shear strength of 240,000 psi for carbon steel music wire.

The comparison of the "rupture moduli" with the tensile or shear strength illustrates that endodontic files and reamers function more ideally in torsion than in bending and that reamers function more ideally in bending than files. The most probable causes for the lack of agreement of the "rupture moduli" in bending and the tensile strength is the use of the section modulus calculated from the geometry of the instruments $3 \mathrm{~mm}$. from the tip and the assumption that this section modulus was constant over the length of the beam. This assumption was more serious in files than in reamers, since the former were more twisted in design.

The agreement between the "rupture modulus" in torsion was again better in reamers than files because the files, which were more tightly twisted, had greater mass. The assumption that the polar section modulus was constant between $3 \mathrm{~mm}$. from the tip and the shank led to a poorer approximation for files than reamers. It is important to note that the endodontic reamer and file, when bound in a root canal, had torsional properties comparable to a blank having a uniform section modulus from the point of bending to the shank. Since bending takes place over a span and torsion takes place in a section, the effect of varying section moduli along the length of the instruments had less effect on the "rupture modulus" in torsion than in bending. There is also some indication that reamers with triangular cross sections function more ideally in bending than those with square cross sections.

\section{Summary}

It was shown that for carbon steel files and reamers the ultimate bending and torsional moments could be estimated from experimental values of the yield point- bending and torsional moments, with excellent agreement for the small and good agreement for the large instruments.

The "rupture moduli" in bending and torsion were calculated from the yield point-bending moments and the section moduli at the position on the instrument where it was gripped. The "rupture moduli" of the instruments in bending did not compare well with the tensile strength, but the "rupture moduli" in torsion compared favorably with the shear strength. The lack of agreement resulted from the tapered sections and the degree of twisting of the instruments. The data indicate that endodontic instruments have almost ideal behavior in torsion but less than ideal behavior in bending.

\section{References}

1. Bucher, J. F. A Preliminary Investigation of the Feasibility of Stainless Steel Files as Root Canal Filing Instruments and as a Root Canal Filling Material. MS thesis, University of Michigan, School of Dentistry, 1958.

2. Hever, M. A. A Study of the Structural, Dimensional and Physical Characteristics of Root Canal Instruments. MS thesis, University of Michigan, School of Dentistry, 1959.

3. Craig, R. G., and Peyton, F. A. Physical Properties of Carbon Steel Root Canal Files and Reamers, Oral Surg., 15:213-226, 1962.

4. Craig, R. G., and Peyton, F. A. Physical Properties of Stainless Steel Endodontic Files and Reamers, Oral Surg., 16:206-217, 1963.

5. Sargent, J. E., and Stemler, J. Torsional Properties of Endodontic Instruments, Microfilm IADR Dental Materials Section, Paper M43, Los Angeles, Calif., March 19-22, 1964.

6. Craig, R. G., McIl wain, E. D., and Peyton, F. A Bending and Torsion Properties of Endodontic Instruments, Oral Surg., In press.

7. Timoshenko, S. Strength of Materials. Part 11 . Advanced Theory and Problems. New York, D. Van Nostrand Co., Inc., 1941.

8. Papov, E. P. Mechanics of Materials. Englewood Cliffs, N. J., Prentice-Hall, 1952, p. 48-54; $94-$ 107.

9. NADAI, A., Theory of Flow and Fracture of Solids. New York, McGraw-Hill, 1950, vol. 1, p. 379526; vol 2, p. 435-506.

10. OBERG, E., and Jones, F. D. Machinery's Hand$b o o k$, ed. 17. New York, Industrial Press, p. 358367. 